\title{
Excision of Oral Submucous Fibrosis and Reconstruction with Full Thickness Skin Graft: A Case Report and Review of Various Treatment Modalities
}

\author{
Jitendra Kumar ${ }^{1 *}$, Pankaj Goel ${ }^{2}$, Vineeta Yadav ${ }^{3}$ and Arif Merchant \\ ${ }^{1}$ Resident Doctor, Department of Dentistry, Saket Nagar, AIIMS Bhopal, Madhya \\ Pradesh, India \\ ${ }^{2}$ Professor and HOD, Department of Dentistry, Saket Nagar, AIIMS Bhopal, Madhya \\ Pradesh, India \\ ${ }^{3}$ Senior Resident, Department of Dentistry, Govt. Medical College, Gondia, \\ Maharashtra, India \\ ${ }^{4}$ Consultant Maxillofacial Surgeon, Dhule, Maharashtra, India \\ *Corresponding Author: Jitendra Kumar, Resident Doctor, Department of Dentistry, \\ Saket Nagar, AIIMS Bhopal, Madhya Pradesh, India.
}

Received: December 17, 2021

Published: January 18, 2022

(C) All rights are reserved by Jitendra Kumar., et al.

\section{Abstract}

The choice of treatment related to Oral Submucous Fibrosis is still controversial and depends on the stage of the clinical and histological finding.

A 25-year male patient was diagnosed with Oral Sub Mucous Fibrosis with the help of histological and clinical finding. The mouth opening of the patient was less than $5 \mathrm{~mm}$. A surgical treatment was planned for the patient by excision of the lesion followed by placement of graft from the abdomen.

The report highlights the disability limitation and treatment plan in severe case of Oral Sub Mucous Fibrosis.

Keywords: Oral Submucous Fibrosis; Oral Cancer; Tertiary Prevention; Disability Limitation

\section{Introduction}

Over decades Oral Sub Mucous Fibrosis has been a major area of concern for clinicians and due to complex etiopathogenesis, the choice of treatment remains controversial [1], the literature put forward a variety of choices for treating these patients, but no single treatment has proven to be very effective and in advances cases the surgical excision of the fibrotic bands and temporalis myotomy along with coronoidectomy followed reconstruction remains the mainstay treatment choice rendering to improved quality of life for the patients [2]. OSMF is a collagen deposition disorder characterized by excessive deposition of collagen and impaired collagen clearance characterized by juxta epithelial inflammatory reaction and fibroelastic changes within the lamina propria leading to decreased vasculature thus reducing blood flow which in turn leads to atrophy of the mucosa, reducing its elasticity and excessive fibrosis [3]. The disease is prevalent in the Asian subcontinent predominantly in regions where areca nut chewing is common. However, few idiopathic cases have also been reported where patients do not present with any positive history of areca nut abuse and genetics remains the culprit, other causes include autoimmunity, vitamin B, $\mathrm{C}$, and iron deficiencies, consumption of spicy foods, and human papilloma virus (HPV) infection [4]. The disease spans various stages, the initial clinical presentation includes a burning sensation of the oral mucosa, often these patients confirm of inability to eat hot and spicy food, As the disease progresses and the formation of circumoral fibrotic bands rendering to the limitation in stomatic opening imparting difficulty in mastication, speech, and swallowing, in later stages patient presents with less or rather no mouth 
Excision of Oral Submucous Fibrosis and Reconstruction with Full Thickness Skin Graft: A Case Report and Review of Various Treatment Modalities

opening and oropharyngeal pain often radiating to the ears [5]. Most of the time these patients ignore mild symptoms and often report to the clinician in advanced stages where symptoms have worsened, the case report aims to highlight the treatment plan for severe cases of Oral Sub Mucous Fibrosis along with the review of the various treatment option. The report was carried using CARE guidelines [6].

\section{Case Report}

A young male patient of 25 years of age reported in the Department of dentistry in a Tertiary care hospital with reduced mouth opening accompanied by severe burning of mouth which has gradually increased for 2-3 years, on enquiring about the addiction and personal habits Patient confronted with a positive history of consuming smokeless tobacco in form of betel quid (areca nut) for the last 8-10 years, multiple times in a day. However, the patient narrated that due to inability to open his mouth he has given away the habit 2-3 months back and has been on liquid and semi-solid diet since then. On examination, we have observed that the patient had minimal mouth opening with an interincisal distance of $<5 \mathrm{~mm}$ (Figure 1), on palpation the patient had severe fibrosis extending from the retromolar region anteriorly forming the circumoral band on both the sides with no malignant changes. We performed fiberoptic examination to assess the soft palate and floor of mouth, it showed that there was no involvement of the soft palate and floor of mouth with an absence of any ulcerative changes. The neck nodes were also examined and found to be non-palpable at all the level. Based on history and our clinical examination the diagnosis of Oral Sub Mucous Fibrosis was made. We explained the surgical option to the patient for which he took a week to decide and rereported a week later for surgery.

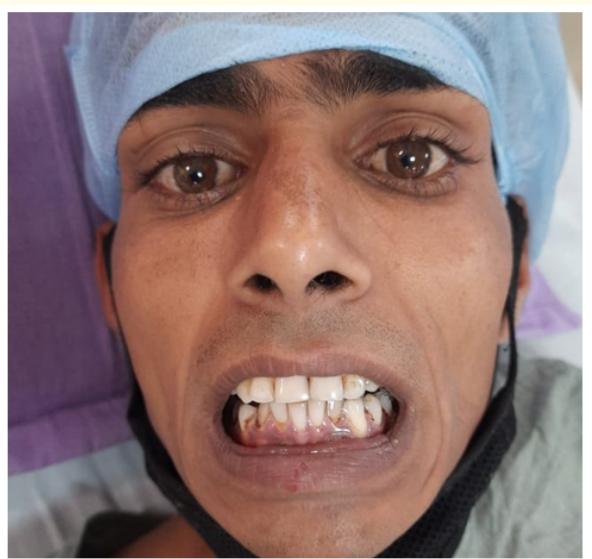

Figure 1: Pre-operative reduced mouth opening.
Surgery was performed under general anesthesia with fiberoptic assisted intubation following the standard surgical protocol. $2 \%$ lidocaine with ADR was infiltrated at the surgical site. An electrocautery device was used for surgical excision of the fibrous bands via transoral approach with a cautious check to not to damage any vital structures followed by B/L temporalis myotomy, a consensus was made among the team and all the third molars were also extracted and on-table mouth opening was assessed and was found to be satisfactory $(40 \mathrm{~mm}$ ) (Figure 2). From the right side of the abdomen full-thickness skin graft was taken, to match the recipient site the shape of the graft was chosen to be oval $10 * 5 \mathrm{~cm}$ (Figure $3)$; post dissection hemostasis was achieved, the donor site was closed with 3-0 silk suture. Graft thus harvested was split into two halves and was sutured B/L respectively with 4-0 vicryl. (Figure 4) On completion, the donor site dressing was done, and a standard antibiotic regimen and post-operative instruction was given to the patient which included physical therapy from $4^{\text {th }}$ day using Heister mouth gag appliance and ice-cream sticks (Figure 5). He was advised to further continue with Heister mouth gag exercise for at least 5 times per day for 4 weeks. Patient was called for follow up after 4 months, mouth opening at $40 \mathrm{~mm}$ with good ability to have a satisfactory masticatory function. A biannual follow up was advised. Consent from the patient was taken for publication of the report.

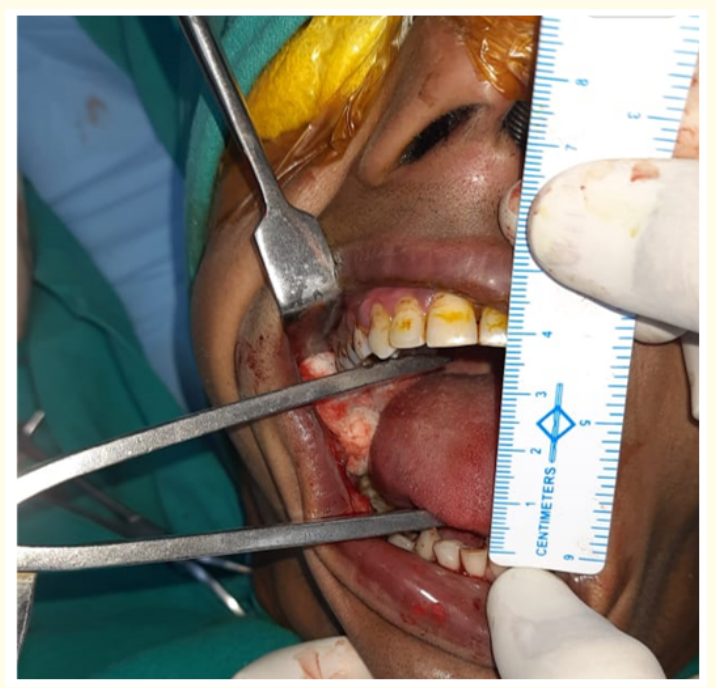

Figure 2: Mouth opening to $40 \mathrm{~mm}$. (Intraoperative). 


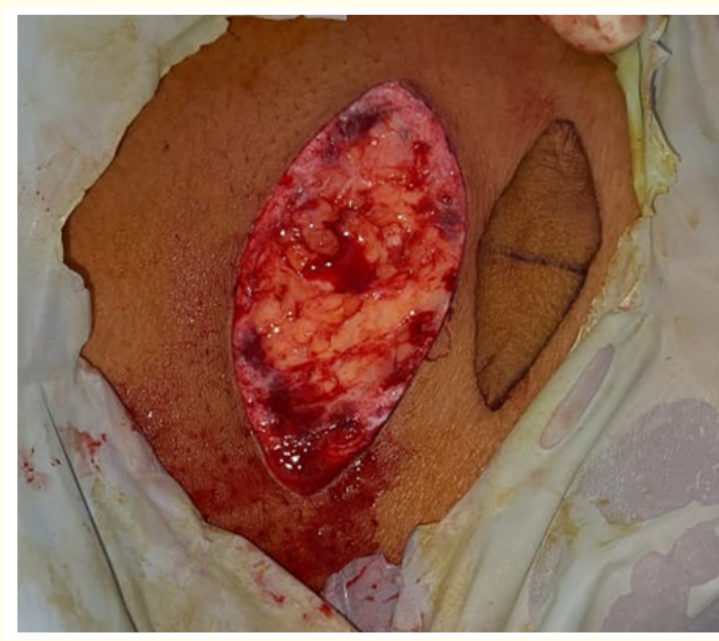

Figure 3: Graft from donor site (Rt. Side Abdomen).

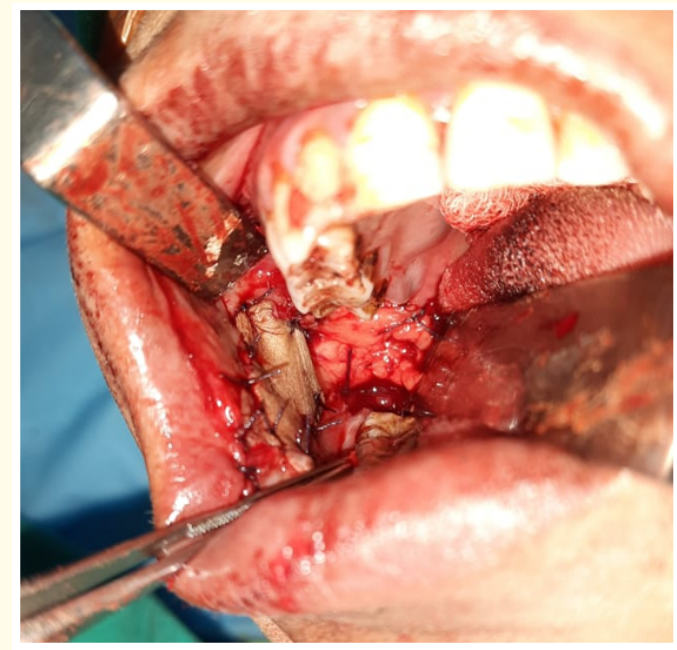

Figure 4: Placement of graft to recipient site.

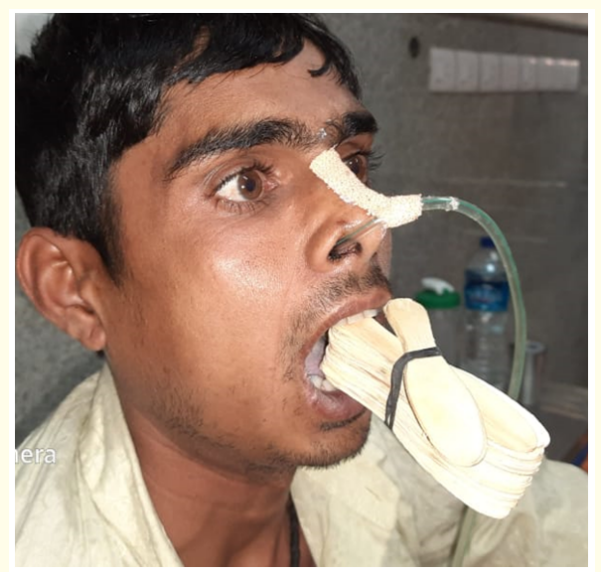

Figure 5: Post-operative.

\section{Discussion}

Treatment of OSMF chiefly aims to treat trismus, decrease burning sensation and restore quality of life. Regarding the high proportion of this condition in our subcontinent, it is important to standardize the treatment protocol for OSMF. The literature is suggestive of various treatment modalities available that include both non-surgical and surgical options. Drug therapy modulates degradation of the extracellular matrix and has an anti-inflammatory action it also blocks fibroblast thus reducing the proliferation and collagen deposition [3]. Corticosteroids such as dexamethasone, methylprednisolone, and betamethasone are synthetic drugs with glucocorticoid-like action and the Intralesional injection substantially improves mouth opening and alleviates the burning sensation. Pentoxifylline has also been tried for the treatment, it is a xanthine derivative primarily used to mitigate muscle pain, the mechanism of action is yet not clear however it is believed that it competitively and non-selectively inhibits phosphodiesterase, suppresses TNF- $\alpha$ production in lipopolysaccharide (LPS)-stimulated human monocytes blocks leukotriene synthesis, and diminishes the inflammatory reaction. Pentoxifylline is found to be helpful in mouth opening and reducing the burning sensation [7]. It also facilitated swallowing and speech. Compounds such as butylidenephthalide, glabridin, asiatic acid, tanshinone, and salvianolic acid B. Butylidenephthalide is potential efficacious against OSMF most of these agents do not have a clear delineation of the mechanism of action and are considered to downregulate $\alpha$-SMA, fibronectin, and type 1 collagen A1 and also reduces myofibroblast bioactivity [8]. Other likely anti-OSMF effective compounds comprise epigallocatechin-3-gallate (EGCG), aloe vera, curcumin, lycopene, and honey, there has been a mixed response to these agents in various clinical trials [9]. Buccal Fat Pad The buccal fat pad as an interposition graft is one of the most practiced techniques by many surgeons across the globe, the technique gives good long-term reliable results in the treatment planning of OSMF [10]. The nasolabial flap is alternatively a popular intervention used in the surgical treatment of OSMF, the literature has a paucity regarding the usage of free flaps for surgical management of OSMF however few articles suggests the usage of Radial forearm flap for the same [11]. The use of SSG is purely supportive and protective in post-operative downtime. Graft material can serve as a coverage layer over the raw area, or it can be used in conjunction with Flaps and serves to protect interposition flaps. Gradually SSG and FDG have been gaining popularity due to the ready availability and being less technique sensitive and can be taken up by less experienced surgeons giving fair results to the patients, the decreased incidence of donor site morbidity also explains the gain in popularity of the same. Results with skin grafting to cover the raw areas has a mixed review in the literature and 
incidence of shrinkage, contracture, and rejection of the graft had been mentioned [12].

\section{Conclusion}

The reports highlight the tertiary prevention in case of severe case of Oral Sub Mucous Fibrosis. The strength of the study is the surgical procedure followed using the abdomen graft. The takeaway message is the disability limitation of the case.

\section{Bibliography}

1. Shih YH., et al. "Oral Submucous Fibrosis: A Review on Etiopathogenesis, Diagnosis, and Therapy". International Journal of Molecular Sciences 20.12 (2019): 2940.

2. Liu D., et al. "A practical guide to the monitoring and management of the complications of systemic corticosteroid therapy". Allergy, Asthma and Clinical Immunology 9 (2013): 30.

3. Tsai CC., et al. "Deficiency in collagen and fibronectin phagocytosis by human buccal mucosa fibroblasts in vitro as a possible mechanism for oral submucous fibrosis". Journal of Oral Pathology and Medicine 28 (1999): 59-63.

4. Arakeri G., et al. "Oral submucous fibrosis: An update on current theories of pathogenesis". Journal of Oral Pathology and Medicine 46 (2017): 406-412.

5. More C., et al. "Classification system of Oral submucous fibrosis". Journal of Indian Academy of Oral Medicine and Radiology 24.1 (2012): 24-29.

6. https://www.care-statement.org/

7. Rajendran R., et al. "Pentoxifylline therapy: a new adjunct in the treatment of oral submucous fibrosis". Indian Journal of Dental Research 17.4 (2006): 190-198.

8. Chan SS., et al. "Mechanisms underlying the vasorelaxing effects of butylidenephthalide, an active constituent of Ligusticum chuanxiong, in rat isolated aorta". European Journal of Pharmacology 537 (2006): 111-117.

9. Hsieh YP., et al. "Arecoline stimulated early growth response-1 production in human buccal fibroblasts: Suppression by epigallocatechin-3-gallate". Head and Neck 37 (2015): 493-497.

10. Khanna JN and Andrade NN. "Oral submucous fibrosis: a new concept in surgical management. Report of 100 cases". International Journal of Oral and Maxillofacial Surgery 24 (1995): 433-439.
11. Lee J-T., et al. "A double-skin paddle radial forearm flap for the reconstruction of oral submucous fibrosis". Tzu Chi Medical Journal 18 (2006): 362-369.

12. Morawetz G., et al. "Oral submucous fibrosis". International Journal of Oral and Maxillofacial Surgery 16 (1987): 609-614.

\section{Assets from publication with us}

- Prompt Acknowledgement after receiving the article

- Thorough Double blinded peer review

- Rapid Publication

- Issue of Publication Certificate

- High visibility of your Published work

Website: www.actascientific.com/

Submit Article: www.actascientific.com/submission.php

Email us: editor@actascientific.com

Contact us: +919182824667

Citation: Jitendra Kumar., et al. "Excision of Oral Submucous Fibrosis and Reconstruction with Full Thickness Skin Graft: A Case Report and Review of Various Treatment Modalities". Acta Scientific Dental Sciences 6.2 (2022): 78-81. 